\title{
PART II
}

\section{CASE-BASED QUALITATIVE TECHNIQUES}

\author{
Professor Peter Allen
}

This section tries to address the underlying question of how far the new ideas of 'complexity' and 'complex systems' might actually improve understanding and outcomes in the social sphere compared to that of well-intentioned common sense? First, it should be admitted that common sense is not necessarily densely scattered in our world, and so the question this section addresses is about the approach and the process of how a method would help us apply 'complex systems thinking' to social situations, with a view to doing better than before.

One of the main thinkers behind the discovery of 'complex systems' was Ilya Prigogine who used the title From Being to Becoming for one of his books, to capture an important difference that complexity and open systems thinking signalled. Instead of considering an understanding of the world in terms of either an equilibrium (possibly expressing some optimum), or as mechanical systems simply churning forward with given interactions, he saw that complexity was really an evolutionary vision. Complex systems, like people are not 'passive'. They are both creative and exploratory themselves, if permitted, and may be embedded in creative and exploratory surroundings as well.

Although the fundamental laws of physics, are always true, they do not provide an explanation of what goes on in the living and social world. Complex systems are multilevel systems with networks, organizations and structures and over time they evolve as some parts wither and others grow, and entirely new aspects can emerge. So, in origami we see that a piece of rectangular paper, when folded, can exhibit emergent characteristics and functions as a result of symmetry breaking in its morphology - which the mechanics of the paper cannot possibly 'explain'. The origami object interacts with its environment entirely differently from a sheet of paper. It can be a bird, a vase, a hat, a box, and all sorts of things. The laws of physics can only tell us about the elasticity and density of the paper, but nothing about the forms and functions that can emerge. The paper and the laws of physics are simply the materials on which the forces of complexity can create all kinds of amazing things. From macro-molecules, this includes living matter and people. And people can also get 'locked in' to existing situations which seem be unfair and very bleak, and not know how to change things.

They are what they are now, but what could they become?

This is where the ideas coming from complexity provide a framework for reflection of 
what could be - becoming instead of just being. Remarkably, the chapters in this section present real cases in which the ideas coming from complexity science shapes their interventions, and leads to some amazingly successful outcomes.

This Handbook is focused particularly on Research Methods in Complexity Science, and this section is very much devoted to the methods that have been created and used in real cases in social science. They share the idea of setting up a method that can be used in different possible situations and circumstances; in order to create an enabling environment for joint reflection on who is who, what are the issues and what might be done to improve things. In the chapter by Eve Mitleton-Kelly this:

Enabling Environment has to be co-created by the people involved in the actual case and those guiding the process. An Enabling Environment is not a 'free for all'. It sets very clear aims and objectives, both in the short and longer term. It also provides an enabling infrastructure with suitable training, support, facilities and so on for local leaders to enable them to respond appropriately and in a timely manner. To work well, the Enabling Environment needs very good communications.

The chapter describes the EMK complexity methodology in sufficient detail to enable researchers to use it and illustrates how part of the approach was used in a case study. The method identifies the multiple dimensions in the problem space and their co-evolutionary dynamics (how the different dimensions and issues interact and change each other in the process). The methodology recognizes that there is no single, simple 'solution' to a complex problem, but that the problem can be addressed effectively by setting up an enabling environment, which directly addresses the co-evolving clusters and evolves with the problem space.

Another important point that is raised in this first chapter is that of different cultural perspectives. In the Indonesian case described by EMK, it is completely unclear what would be a 'good' outcome for the many different players. The underlying issue is deforestation, and we might think that it is simply a question of reducing deforestation. But locally there may be very complex relationships and loyalties that come into play, and complex social relations and loyalties will affect what decisions may seem 'good' for different participants. The customs of the culture may appear to us to resemble nepotism and corruption, but all we can do is to provide an 'enabling arena' of discussion, so that over time perhaps they may choose to modify such practices. The chapter points out that it is important to be aware of these differences in culture and to have the courage to articulate the distinction between what was culturally acceptable and what amounted to corruption, when money changed hands. But this is really all part of the 'becoming' and there is no clear evidence of how long it may take. We can perhaps only contribute to the discussion of different aspects, hoping that some of the more glaring problems can be partially reduced, and perhaps changed more in the future.

The chapter contributed by Lesley Kuhn describes some creative applications of a complexity informed social research. A complexity perspective suggests that the only viable long-term strategy for the development of a complex social system, such as the Mount Druitt community, an example used in the chapter, is for the system to learn about itself. Informing key or relevant people is a powerful way of nudging complex social systems towards self-managed improvement.

A complexity informed approach that corresponds to a depiction of life as selforganizing, dynamic and emergent is well suited to inquiry into human experience as it 
brings a sense of openness to appreciating and studying human experience as it emerges. The outcome from complexity informed social research is that the researcher, the situation and the theory are adaptively engaged and simultaneously improved. The chapter introduces some fairly new ideas (for me) such as: vortical postmodern ethnography, which can be seen as having five main parts. First it focuses on narratives, discourse and behaviour as these unfold during the research. Second, it relies on qualitative data in the form of formal and reflective narratives pertaining to the research. Third, it takes a holistic perspective that sees the research as being focused on one level, but implicating issues at higher and lower levels. Fourth, expects new ideas, insights, hypotheses and questions to arise from the vorticity generated by the research process. Fifth, analysing data for identification of themes that have a strong resonance for participants and as treating data generation as a further source of vorticity that leads to new cycles of emergence. Also, as an ex-physicist I find the invention of phrase space as some kind of social version of the phase space of physics, rather interesting. They might represent the space of the dimensions of the whole problem.

The chapter then illustrates the application of these ideas to a community in New South Wales, Australia, in Mount Druitt, an area that is culturally and linguistically diverse, with a large Indigenous population and low median income. The program was begun in response to citizen requests for assistance in developing leaders and involved participants who were: engaged in the community in some way (paid or voluntary); recommended by a community organization, government agency or religious organization; between 15 and 90 years of age; committed to Mount Druitt. Once again the intervention seems to have had extremely beneficial effects on the community, and demonstrates the potential importance of these 'complexity' related approaches.

In the chapter by Hazel Stutely and Jonathan Stead we find an approach which is also inspired by complexity thinking. The Connecting Communities (C2) project has developed its own seven-step application of complexity science to underpin a delivery framework. C2 offers an intervention, delivered on site by a small team of experienced practitioners, working alongside local residents and service providers to enable them to implement the framework. "It placed great value on widespread connectivity, the creation of new relationships and dialogue based on trust. Conversations, humility and respect, I now realised, contributed hugely to the creation of that all-important enabling environment, which released the resourcefulness of this community to become self-organising and achieve such significant and dramatic outcomes." These ideas had been tried out initially in the Beacon project set in a severely disadvantaged social housing estate (pop. 6,000 ) in Falmouth. The results were in my view staggering: overall crime rate down 50 per cent; unemployment down 71 per cent; improvement to 1,000 homes; educational attainment up 100 per cent; child protection rates down 42 per cent; post-natal depression down 70 per cent; childhood asthma down 50 per cent; teenage pregnancy zero! Other projects have followed. REACH (Redruth Enabling Active Community Health) 2004-2006, is an example of close collaboration between a community and the emergency services. Outcomes included: 210 patients treated between 2004 and 2006 on site; 30 per cent drop in incidence of under-age problem drinking and an 18 per cent reduction in emergency call outs.

Since then, the approach has been used in a series of further projects: OPERATION GOODNIGHT: with the police and residents, in Redruth in 2008. More recently the 
Greenfingers project was run in partnership with Duchy Agricultural College. It was highly successful and has transformed not only estate gardens and green spaces but the lives of its participants who have accessed further education in greater numbers. The TR14ers Camborne: Cornwall 2005-2008: It was founded in response to significant police concerns about rising levels of antisocial behaviour (ASB) and health inequalities affecting the youth of Camborne. The majority of young people that attend the TR14ers live on remote social housing estates with little social or play facilities and their families are often troubled by a raft of health and socio-economic issues. The project has led to a large drop in antisocial behaviour, and to much improved health and educational outcomes.

The improvements in the lives of underprivileged communities that these projects have attained are truly remarkable.

In the next chapter, Robin Durie, Craig Lundy and Katrina Wyatt use complexity principles to understand the nature of relations for creating a culture of publicly engaged research within Higher Education Institutes. The chapter presents an approach to social systems which is based on the complex systems approach, and the behaviour of complex adaptive systems. That is, the behaviour of the parts of complex systems results from a co-evolution with the other parts. Similarly, the behaviour of the whole system is affected by its co-evolution with the environment of which it forms a part. Thus, complex adaptive systems are continually responding and adapting to changes in their environment, just as the environment itself changes and adapts to changes amongst its elements. The ongoing behaviour of the whole system, as well as of its parts, remains to a greater or lesser degree unpredictable; such novel, unpredictable, behaviours - often a consequence of the 'selforganization' of the system - are thus said to be 'emergent'.

The chapter discusses the reasons why it makes sense to use a complexity approach to public engagement in research. It offers the potential for comparing phenomena such as networks, sustainability and resilience in biological systems with similar phenomena in social systems, and thereby opens the possibility of transferable co-learning about the causes of such phenomena. It provides a broader framework for ideas and confirmation than just the actual examples studied. In particular, it looks at notions such as coevolution which seem appropriate for the way that relations between communities and researchers may develop. The principle of emergence offers a potentially insightful means of capturing the tendency for community engagement to yield novel and unexpected research outcomes. Most of all, the potential for phenomena such as co-evolution, selforganization and emergence stems, according to complexity theory, from the distinctively nonlinear nature of the dynamic relations between agents, and this focus on dynamic relational processes appears to offer a particularly effective means for making sense of the relations that underpin research projects involving community-university partnerships.

In this section on methodology of interventions the chapter then offers a three level method of analysis. An initial analysis records interviews and focus groups, which are then transcribed and checked. A number of cross-cutting themes are identified, and these are presented during 'negotiated feedback' sessions. The resulting discussions lead to the refinement of the cross-cutting themes. Then there is a complexity analysis. This is a secondary analysis of the emergent cross-cutting themes from the perspective of complexity. Our provisional assumption here is that the cross-cutting themes should exemplify principles pertaining to complex systems. The third phase is the critical-reflective analysis. This is a rigorous interrogation of the complexity themes involving the lead researcher 
and Robin Durie and Katrina Wyatt. The purpose of this final iteration of analysis was to critically reflect on the appropriateness of the use of complexity theory as an interpretative framework for the primary data, and at the same time to reflect on whether this bringing together of the first two levels of analysis poses questions of complexity theory itself, and of its applicability to social systems in general, and systems of community-university research partnerships in particular.

There is an important issue that the chapter also highlights. It is not about the research carried out, but is about the relations between the people being studied and the researchers doing it. In the projects here, there had been a commitment to 'stay the distance' from the outset, and this helped greatly in creating the kind of relationships that lead to successful engagement. It is an important part of an 'enabling environment' for these projects. Community participants were concerned about researchers just coming in to 'do' the project, tick their boxes and then leave, without having a real commitment to the outcome. Fortunately, many academic partners recognized this issue as important in gaining the trust of communities. This was seen to be an important step in the creation of meaningful community-academic relations. This raises the problem of the short-term nature of research funding in this kind of domain.

The research projects sought to identify patterns of enabling behaviours for successful community engagement in research. It also sought to undertake a complexity informed case study approach to try and identify patterns of dynamic processes by which successful engagement occurs. A multi-phased framework was developed aimed at understanding the distinctive dynamics involved in the processes constituting the engagement cycle. The perspective of complexity theory suggests that the focus for community engagement with academic research should be on the dynamic relations that constitute the processes of engagement, and, in particular, on how such relations might emerge, or be co-created, within engagement processes.

This short introduction to Part II of the Handbook focuses on some the chapters that describe research methods being developed to look at social systems using the perspective of complexity thinking. The different chapters included provide clear descriptions of methods based on complexity thinking, and provide an optimistic view of what has been happening, and how the approaches should be further developed. Clearly, the way that complex systems evolve, co-evolve and change qualitatively over time is of great relevance and importance for our understanding of social systems. These applications move us away from the 'laissez faire' approach to neighbourhoods and society, and show us that we can help people to help themselves improve their communities, their health and their lives by using the evolutionary model offered by complex systems thinking.

\section{REFERENCE}

Prigogine, I. (1980), From Being to Becoming: Time and Complexity in the Physical Sciences, New York: W.H. Freeman \& Company. 
Professor Peter Allen - 9781785364426 